\title{
Adiposity Measurements by BMI, Skinfolds and Dual Energy X-Ray Absorptiometry in relation to Risk Markers for Cardiovascular Disease and Diabetes in Adult Males
}

\author{
Ahmad A. Hariri, ${ }^{1}$ Nicholas S. Oliver, ${ }^{1}$ Desmond G. Johnston, ${ }^{1}$ \\ John C. Stevenson, ${ }^{2}$ and Ian F. Godsland ${ }^{1,3}$ \\ ${ }^{1}$ Division of Diabetes, Endocrinology and Metabolic Medicine, Department of Medicine, Faculty of Medicine, \\ Imperial College London, St Mary's Campus, Norfolk Place, London W2 1PG, UK \\ ${ }^{2}$ National Heart \& Lung Institute, Imperial College London, Dovehouse Street, London SW3 6LY, UK \\ ${ }^{3}$ Wynn Reader in Human Metabolism, Endocrinology and Metabolic Medicine, Imperial College London, St. Mary's Hospital, \\ G1 Medical School Building, Norfolk Place, London W2 1PG, UK
}

Correspondence should be addressed to Ian F. Godsland; i.godsland@imperial.ac.uk

Received 12 June 2013; Revised 2 October 2013; Accepted 3 October 2013

Academic Editor: Robert Pichler

Copyright (C) 2013 Ahmad A. Hariri et al. This is an open access article distributed under the Creative Commons Attribution License, which permits unrestricted use, distribution, and reproduction in any medium, provided the original work is properly cited.

Background. Choice of adiposity measure may be important in the evaluation of relationships between adiposity and risk markers for cardiovascular disease and diabetes. Aim. We explored the strengths of risk marker associations with BMI, a simple measure of adiposity, and with measures provided by skinfold thicknesses and dual energy X-ray absorptiometry (DXA). Subjects and Methods. We evaluated in three subgroups of white males $(n=156-349)$, participating in a health screening program, the strengths of relationship between measures of total and regional adiposity and risk markers relating to blood pressure, lipids and lipoproteins, insulin sensitivity, and subclinical inflammation. Results. Independent of age, smoking, alcohol intake, and exercise, the strongest correlations with adiposity measures were seen with serum triglyceride concentrations and indices of insulin sensitivity, with strengths of association showing little difference between BMI and skinfold and DXA measures of total and percent body fat $(R=0.20-0.46, P<0.01)$. Significant but weaker associations with adiposity were seen for serum HDL cholesterol and only relatively inconsistent associations with adiposity for total and LDL cholesterol and indices of subclinical inflammation. Conclusions. BMI can account for variation in risk markers in white males as well as more sophisticated measures derived from skinfold thickness measurements or DXA scanning.

\section{Introduction}

The relationships between obesity and cardiovascular disease (CVD) and type 2 diabetes mellitus (T2DM) are currently understood in terms of systemic changes that excess adipose tissue can induce in the physiologic and metabolic risk markers for these diseases. Adipose tissue products are involved in the pathogenesis of essential hypertension [1]; moreover, as an endocrine organ, through its release of various adipokines, adipose tissue can influence the transport and metabolism of lipids and lipoproteins $[2,3]$, glucose metabolism, and insulin sensitivity $[4,5]$ and can promote subclinical inflammation [6]. Variation in regional adipose tissue distribution may significantly affect risk markers for T2DM and CVD and risk of these diseases, with stronger associations for central obesity than for generalised obesity [7-12].

Elucidation of relationships between adiposity and physiologic and metabolic variables is important for our understanding of the role of increasing adiposity in health and disease, and evaluation of relationships between adiposity and risk markers is an important aspect of CVD and T2DM risk evaluation. Choice of adiposity measure is clearly a consideration in such investigations, and this can be informed by knowledge of the strength of relationship between a given adiposity measure and established CVD and T2DM risk markers. Therefore, it is important to establish 
whether simpler measures of adiposity can provide as much information as measures that are more complex and more theoretically rigorous. Here, we test the hypothesis that, in a cohort restricted to white males, body mass index (BMI), a frequently used, simple measure of net adiposity, will correlate with risk markers relating to blood pressure, lipid and lipoprotein metabolism, insulin sensitivity, and subclinical inflammation as strongly as more sophisticated measures. These include a measure of body fat mass derived from four skinfold thickness measures and direct measures of total and regional fat mass derived using dual energy Xray absorptiometry (DXA).

\section{Subjects and Methods}

2.1. Design. The Heart Disease and Diabetes Risk Indicators in a Screened Cohort (HDDRISC) study is an open cohort study of 1192 white males recruited as part of a company health screening program. The study began in 1971 and data collection ended in 2000. Skinfold thicknesses were measured from the beginning of the study to 1996 and DXA body fat masses from 1989 to the end of the study. The majority of those who had skinfolds measured underwent an oral glucose tolerance test (OGTT) and a minority underwent an intravenous glucose tolerance test (IVGTT). Among those who had DXA measurements of body fat mass, very few underwent an OGTT, but the majority underwent an IVGTT.

The present analysis concerns three bodies of data derived from the HDDRISC study. Each dataset included participants who were nondiabetic (fasting plasma glucose, FPG, $<7.0 \mathrm{mmol} / \mathrm{L}$ ) and not grossly obese $\left(\mathrm{BMI}<35.0 \mathrm{Kg} \cdot \mathrm{m}^{-2}\right.$, corresponding to $3 \mathrm{SD}$ above the mean for baseline $\mathrm{BMI}$ measurements in the entire cohort) and received measurements of BMI, blood pressure, fasting serum total, low-density lipoprotein (LDL) and high-density lipoprotein (HDL) cholesterol, triglycerides, fasting plasma glucose and insulin, and laboratory measures related to inflammation. Datasets were then distinguished as follows: (1) the 349 men who received measurement of skinfold thicknesses and assessment of insulin sensitivity by OGTT ("BMI/SF group" studied between 1972 and 1991); (2) the 269 men who received measurement of DXA fat masses and assessment of insulin sensitivity by IVGTT ("BMI/DXA group" studied between 1989 and 1997); (3) the 156 men who received measurement of both skinfold thicknesses and DXA fat masses and assessment of insulin sensitivity by IVGTT ("BMI/SF/DXA group" studied between 1989 and 1996).

2.2. Participants. The majority of participants in the HDDRISC study group were senior executives of a large company. They were generally healthy and in employment or recently retired. The study received local ethics committee approval, and each participant gave their written informed consent.

2.3. Procedures. All participants fasted overnight (only water) and refrained from smoking and alcohol prior to attending a dedicated metabolic ward. Here, a full medical history was taken, including details of smoking habits, alcohol intake, and exercise behaviour.

Weight and height were measured in light clothing without shoes. Among those receiving skinfold measurements, skinfold thicknesses were then measured using the procedure of Durnin and Womersley [13]. Briefly, three measurements were taken, using Harpenden callipers, at biceps, triceps, iliac, and subscapular sites. All readings were measured to the nearest $\mathrm{mm}$, unless values were particularly low $(\leq 5 \mathrm{~mm})$ in which case the nearest $0.5 \mathrm{~mm}$ was recorded. Among those undergoing measurement of total and regional body fat tissue by DXA, scans were carried out on a Lunar Radiation Corporation (Madison, WI, USA) DPX scanner [14] with total body scanning carried out at a transverse speed of $16 \mathrm{~cm} / \mathrm{s}$ or $8 \mathrm{~cm} / \mathrm{s}$ in men who weighed $>90 \mathrm{~kg}$, with subjects receiving radiation doses of 0.05 or $0.1 \mu \mathrm{Gy}$, respectively [15]. Neither waist nor hip circumferences were measured during this study.

After 15-minute rest, systolic and diastolic blood pressures (BP) were taken using a mercury sphygmomanometer. Then, with participants in a semirecumbent position, an indwelling cannula was inserted in the antecubital vein of the nondominant arm for blood sampling. Blood samples were taken for routine haematology and clinical biochemistry measurements, including white blood cell count (WBC), erythrocyte sedimentation rate (ESR), serum globulin and albumin concentrations, serum lipid and lipoprotein concentrations (cholesterol, triglycerides, and HDL cholesterol), and plasma glucose and insulin concentrations (FPG and FPI, resp.). A second sample for FPG and FPI concentration measurements was then taken five minutes later. Either an OGTT or IVGTT was then carried out. For the OGTT, a glucose load of $1 \mathrm{~g} / \mathrm{kg}$ body weight was given, with plasma glucose and insulin measured at 30,60, 90, 120, 150, and 180 minutes, following consumption of the glucose solution. For the IVGTT, as previously described [16], a second cannula was inserted, in this case in the antecubital vein in the arm opposite to the sampling arm, through which a glucose load of $0.5 \mathrm{~g} / \mathrm{kg}$ body weight was given as 50 percent dextrose. Blood sampling was at $3,5,7,10,15,20,30,45,60,75$, $90,120,150$, and 180 minutes following injection of glucose, and there was no augmentation of accompanying insulin concentrations.

2.4. Laboratory Measurements. Routine hematology included measurement of WBC. ESR was measured by the Westergren method. Routine biochemistry included measurement of serum globulin and albumin concentrations. These measurements were made using standard laboratory methodology in our own laboratories or local hospital chemical pathology laboratories. Plasma glucose and insulin and serum total, HDL cholesterol, and triglyceride concentrations were measured in our own laboratories as described previously [17]. Quality control was monitored with commercially available lyophilized sera and by participation in national schemes. Between-batch assay coefficients of variation were plasma glucose $<3 \%$; plasma insulin $<6 \%$; serum cholesterol and triglycerides $<2 \%$; serum HDL cholesterol $<4 \%$; serum globulin and albumin concentrations $<6 \%$. Throughout the 
course of the HDDRISC study, changes in measurement methodology were accompanied by detailed comparisons between methods, with application of correction factors if necessary to maintain continuity of measurement standardization. On completion of data collection for the study, detailed checks were also made to exclude the possibility of laboratory drift significantly affecting results.

2.5. Data Analysis. BMI was calculated as the weight $(\mathrm{kg}) /$ height $(\mathrm{m})^{2}$. The mean of the three skinfold thickness measurements was calculated, and percent body fat (SF \%fat) was calculated from the individual means for the four skinfold thickness measures, according to the method of Durnin and Womersley [13]. Body fat mass (SF total fat) was then calculated as body weight $\times(\mathrm{SF} \%$ fat $/ 100)$. The DXA-derived measurement of total body fat mass (DXA total fat) was expressed as a percentage of body weight (DXA \%fat). Proprietary software was used to distinguish upper body or android fat (DXA android) and lower body or gynoid fat (DXA gynoid) in the DXA scan. The region representative of android fat was defined by an upper horizontal border underneath the chin with vertical borders passing down beside the ribs and a lower border formed by diagonal lines passing through the hip joints and meeting at the perineum. The region representative of gynoid fat was defined as the tissue below these diagonal lines. Android or gynoid fat was expressed as a percentage of DXA total fat (DXA \% android and DXA \% gynoid, resp.). The CV for DXA fat was $1.8 \%$, while CVs for the regional measurements were all $<5 \%$ [18].

Current cigarette smoking was categorised as $0,<5,5-$ $14,15-24$, and $>24$ cigarettes per day alcohol intake as 0 , $<28,28-56$, and $>56$ units per week and exercise habit as no regular, regular nonaerobic, and regular aerobic exercise. Serum low density lipoprotein (LDL) cholesterol concentration was calculated by the Friedewald method [19]. FPG and FPI were taken as the mean of the two fasting measurements. The homeostasis model assessment index of insulin resistance (HOMA-IR) derived from FPG and FPI was calculated [20]. An OGTT-derived index of insulin sensitivity was provided by the Matsuda index (Matsuda$\mathrm{Si}$ ) [21]. An IVGTT-derived measure of insulin sensitivity, $\mathrm{Si}$, (IVGTT-Si) was provided by minimal model analysis of IVGTT glucose and insulin concentrations, implemented according to an optimised algorithm, as described previously [16].

2.6. Statistical Analysis. All data was analysed using STATA 8 for Windows (Stata, College Station, TX, USA). Summary measures were median and interquartile range for continuous variables and percentages for categorical variables. For parametric analyses, measures were square root or log, transformed as appropriate to normalize their distributions. ANOVA for continuous variables and chi-square for categorical variables were used to identify significant variation in BMI and risk marker measures between the groups studied. Dummy variables were assigned for each cigarette smoking, alcohol intake, and exercise category. Relationships between adiposity measures and between each adiposity measure and each risk marker were explored by partial correlation, taking into account covariation with age, cigarette smoking, alcohol intake, and exercise habit. A significance level of $P<0.05$ was adopted with no correction for multiple testings, each correlation undertaken being strongly weighted by existing evidence, thus rendering the universal null hypothesis inapplicable [22]. These analyses were repeated with exclusion of measurements lying $>3 \mathrm{SD}$ outside the mean (theoretically excluding $0.3 \%$ of the data) to minimise any leverage due to outliers. The magnitude and differences in the strengths of the relationships between measures of total body fat and risk markers were explored by comparing confidence intervals for regression coefficients derived from regression analyses incorporating age, cigarette smoking, alcohol intake, and exercise.

\section{Results}

Participant characteristics in each of the three groups are summarised in Table 1. Ages ranged between 26 and 79 years and BMI between 18.4 and $34.9 \mathrm{~kg} / \mathrm{m}^{2}$. Of the $349 \mathrm{men}$ in the BMI/SF group, 65 percent were nonsmokers and 55 percent had an alcohol intake less than $28 \mathrm{U} / w k, 1.2$ percent were taking blood pressure-lowering agents and 0.3 percent lipidlowering agents. There was significant variation between the groups in age $(P<0.001)$, those in the BMI/SF group having a median age of 47.2 years in contrast with 49.4 in the $\mathrm{BMI} / \mathrm{DXA}$ group and 54.2 in the BMI/SF/DXA group. There was also significant variation in BMI $(P<0.001)$ although median BMI in the three groups only varied between 25.1 and $25.5 \mathrm{Kg} \cdot \mathrm{m}^{-2}$. Smoking $(P<0.001)$, alcohol intake $(P<$ $0.001)$, and exercise $(P<0.01)$ also varied between the groups, with heavier smoking, greater alcohol intake, and more exercise evident in the BMI/SF group than in the BMI/ DXA or BMI/SF/DXA groups. Blood pressures $(P<0.05)$ and serum triglyceride concentrations $(P<0.001)$ were higher in the BMI/SF group, and, on the basis of higher levels of ESR and globulin and lower albumin levels $(P<0.001)$, there was evidence of greater inflammation in the BMI/SF group.

3.1. Relationships between Adiposity Measures. BMI was strongly associated with SF total fat, SF \%fat, DXA total fat, and DXA \%fat (partial correlations 0.68-0.84, $P<$ 0.001 ). BMI was also strongly associated with DXA android and gynoid fat (partial correlations 0.68-0.81, $P<0.001$ ). Somewhat weaker associations were seen between BMI and individual skinfold thicknesses (partial correlations 0.37$0.69, P<0.001$ ), and the weakest associations were seen between BMI and DXA \% android and DXA \% gynoid fat (partial correlations 0.30 and -0.30 , resp., $P<0.001$ ). DXA $\%$ android and DXA \% gynoid fat were inversely correlated with an $R$ value of $-0.91(P<0.001)$. Adiposity measures lying outside $3 \mathrm{SD}$ from the mean from these analyses made only very minor differences to the associations observed.

3.2. Relationships between Risk Factors and Adiposity Measures. Our three study groups represent three samples of relationships between BMI and risk factors and two samples of relationships between skinfold thickness-derived measures and DXA-derive measures and risk factors. Relative 
TABLE 1: Study group characteristics.

\begin{tabular}{|c|c|c|c|c|}
\hline & $\begin{array}{l}\mathrm{BMI} / \mathrm{SF} \\
n=349\end{array}$ & $\begin{array}{c}\mathrm{BMI} / \mathrm{DXA} \\
n=269\end{array}$ & $\begin{array}{c}\mathrm{BMI} / \mathrm{SF} / \mathrm{DXA} \\
n=156\end{array}$ & $\begin{array}{c}\text { ANOVA or chi } \\
\text { square } \\
\text { significance }\end{array}$ \\
\hline Age (yr) & $47.2(41.6-52.1)$ & $49.4(42.8-57.0)$ & $54.2(43.1-60.6)$ & $<0.001$ \\
\hline $\mathrm{BMI}\left(\mathrm{kg} / \mathrm{m}^{2}\right)$ & $25.2(23.6-26.8)$ & $25.5(24.2-27.3)$ & $25.1(23.8-26.7)$ & $<0.001$ \\
\hline Smoking (cigarettes per day \%) & & & & $<0.001$ \\
\hline Nonsmoker & 65 & 82 & 79 & \\
\hline$<5$ & 20 & 9 & 10 & \\
\hline $5-14$ & 3 & 6 & 6 & \\
\hline $15-24$ & 6 & 3 & 4 & \\
\hline$>24$ & 6 & 1 & 1 & \\
\hline Alcohol (units/week \%) & & & & $<0.001$ \\
\hline Never drinks & 2 & 2 & 3 & \\
\hline$<28$ & 55 & 75 & 73 & \\
\hline $28-56$ units/week & 33 & 21 & 21 & \\
\hline$>56$ units/week & 10 & 2 & 3 & \\
\hline Exercise (\%) & & & & $<0.007$ \\
\hline No exercise & 39 & 43 & 49 & \\
\hline Nonaerobic & 47 & 42 & 42 & \\
\hline Aerobic & 14 & 15 & 9 & \\
\hline \multicolumn{5}{|l|}{ Drugs (\%) } \\
\hline Lipid lowering & 0.3 & 1.9 & 2.6 & 0.05 \\
\hline Blood pressure lowering & 1.2 & 6.0 & 7.7 & 0.001 \\
\hline SF fat $(\mathrm{Kg})$ & $19.6(16.1-22.9)$ & - & $20.7(17.3-25.0)$ & - \\
\hline DXA fat $(\mathrm{Kg})$ & - & $19.6(16.5-24.9)$ & $18.5(15.8-22.2)$ & - \\
\hline SF $\%$ fat & $24.5(20.9-27.3)$ & - & $25.8(22.6-29.4)$ & - \\
\hline DXA \% fat & - & $24.2(21.4-28.8)$ & $23.3(20.3-26.6)$ & - \\
\hline SF triceps $(\mathrm{mm})$ & $10.9(8.5-13.4)$ & - & $10.5(8.9-12.8)$ & - \\
\hline SF biceps (mm) & $6.1(4.9-8.0)$ & - & $6.1(5.0-7.8)$ & - \\
\hline SF subscapular (mm) & $16.9(13.3-20.0)$ & - & $16.6(13.6-21.3)$ & - \\
\hline SF iliac (mm) & $15.0(10.7-20.0)$ & - & $18.7(13.5-24.4)$ & - \\
\hline DXA android (Kg) & - & $10.6(8.4-13.8)$ & $9.5(7.8-12.0)$ & - \\
\hline DXA gynoid (Kg) & - & $6.1(5.2-7.5)$ & $6.0(5.2-7.2)$ & - \\
\hline DXA \% android (\%) & - & $54.0(50.1-57.5)$ & $51.5(47.4-55.0)$ & - \\
\hline DXA \% gynoid (\%) & - & $31.9(28.6-35.0)$ & $33.2(30.9-36.0)$ & - \\
\hline Systolic BP (mm Hg) & $125(115-135)$ & $120(110-135)$ & $120(110-140)$ & 0.6 \\
\hline Diastolic BP (mm Hg) & $80(70-90)$ & $80(70-85)$ & $80(70-85)$ & 0.01 \\
\hline Cholesterol (mmol/L) & $5.5(4.8-6.3)$ & $5.3(4.8-6.0)$ & $5.2(4.7-5.8)$ & 0.01 \\
\hline LDL cholesterol (mmol/L) & $3.6(2.9-4.2)$ & $3.4(2.9-4.0)$ & $3.3(2.9-3.9)$ & 0.1 \\
\hline Triglycerides (mmol/L) & $1.23(0.93-1.70)$ & $1.16(0.82-1.76)$ & $1.06(0.72-1.51)$ & $<0.001$ \\
\hline HDL cholesterol (mmol/L) & $1.30(1.12-1.51)$ & $1.25(1.08-1.46)$ & $1.27(1.07-1.48)$ & 0.07 \\
\hline FPG (mmol/L) & $5.3(5.0-5.6)$ & $5.3(5.0-5.6)$ & $5.3(5.1-5.6)$ & 0.02 \\
\hline $\mathrm{FPI}(\mathrm{mU} / \mathrm{L})$ & $10.5(6.0-15.5)$ & $9.5(6.5-13.6)$ & $10.0(7.0-14.3)$ & 0.1 \\
\hline HOMA-IR & $2.4(1.5-3.8)$ & $2.3(1.5-3.3)$ & $2.4(1.6-3.4)$ & 0.1 \\
\hline Matsuda-Si & $4.6(3.0-6.9)$ & - & - & - \\
\hline IVGTT-Si (/min/mU/L) & - & $3.2(2.3-4.5)$ & $3.2(2.3-4.4)$ & - \\
\hline
\end{tabular}


TABLE 1: Continued.

\begin{tabular}{|c|c|c|c|c|}
\hline & $\begin{array}{l}\mathrm{BMI} / \mathrm{SF} \\
n=349\end{array}$ & $\begin{array}{c}\mathrm{BMI} / \mathrm{DXA} \\
n=269\end{array}$ & $\begin{array}{c}\mathrm{BMI} / \mathrm{SF} / \mathrm{DXA} \\
n=156\end{array}$ & $\begin{array}{c}\text { ANOVA or chi } \\
\text { square } \\
\text { significance }\end{array}$ \\
\hline WBC $\left(10^{9} / \mathrm{L}\right)$ & $5.5(4.7-6.5)$ & $5.3(4.5-6.2)$ & $5.2(4.5-6.2)$ & 0.1 \\
\hline ESR (min) & $5(2-10)$ & $3(2-6)$ & $3(2-7)$ & $<0.001$ \\
\hline Globulin (g/L) & $24(22-27)$ & $22(20-24)$ & $22(20-24)$ & $<0.001$ \\
\hline Albumin $(\mathrm{g} / \mathrm{L})$ & $42(40-44)$ & $45(43-47)$ & $44(43-46)$ & $<0.001$ \\
\hline
\end{tabular}

Medians (interquartile ranges) for continuous variables and group percentages for categorical variables are shown.

BMI: body mass index; SF: skinfold thickness; DXA: dual energy X-ray absorptiometry; BP: blood pressure; LDL: low density lipoprotein; HDL: high density lipoprotein; FPG: fasting plasma glucose; FPI: fasting plasma insulin; IVGTT-Si: intravenous glucose tolerance test insulin sensitivity; HOMA-IR: homeostasis model assessment of insulin resistance; WBC: white blood cell count; ESR: erythrocyte sedimentation rate.

TABLE 2: BMI/SF $(n=349)$. Partial correlation coefficients and significances between risk marker and body fat measures, independent of age, smoking, alcohol intake, and exercise habit.

\begin{tabular}{|c|c|c|c|c|c|c|}
\hline & BMI & SF fat & SF triceps & SF biceps & SF sub-scapular & SF iliac \\
\hline Systolic BP & 0.05 & 0.09 & 0.10 & 0.06 & $0.12^{*}$ & 0.10 \\
\hline Diastolic BP & $0.13^{\#}$ & $0.14^{*}$ & $0.13^{*}$ & $0.15^{\#}$ & $0.18^{\#}$ & $0.19^{\S}$ \\
\hline Triglyceride & $0.24^{\S}$ & $0.20^{\#}$ & $-0.14^{*}$ & $0.24^{\S}$ & $0.21^{\S}$ & $0.17^{\#}$ \\
\hline HDL Cholesterol & $-0.15^{\#}$ & $-0.12^{*}$ & -0.10 & -0.11 & $-0.16^{\#}$ & -0.05 \\
\hline FPG & $0.17^{\#}$ & 0.06 & 0.02 & 0.09 & 0.08 & 0.05 \\
\hline FPI & $0.18^{\#}$ & $0.19^{\#}$ & 0.10 & $0.14^{*}$ & $0.14^{*}$ & $0.13^{*}$ \\
\hline HOMA-IR & $0.20^{\#}$ & $0.19^{\#}$ & 0.10 & $0.15^{\#}$ & $0.15^{\#}$ & $0.13^{*}$ \\
\hline Matsuda-Si & $-0.33^{\S}$ & $-0.29^{\S}$ & $-0.14^{\#}$ & $-0.25^{\S}$ & $-0.24^{\S}$ & $-0.20^{\S}$ \\
\hline ESR & $0.11 *$ & 0.07 & 0.05 & 0.10 & 0.07 & 0.05 \\
\hline
\end{tabular}

Significances: ${ }^{*} P<0.05 ;{ }^{\#} P<0.01 ;{ }^{\S} P<0.001$.

DXA: dual-energy X-ray absorptiometry; SF: skinfold thickness.

strengths of association according to each adiposity measure are only considered for those risk factors for which each sample showed a significant relationship. Associations for SF and DXA percent fat and risk factors did not differ from those for SF total and DXA total fat and risk factors. Associations between adiposity measures and total and LDL cholesterol, WBC, globulin, and albumin only exhibited isolated significances that were inconsistent between samples. These associations are not considered further. Measures lying outside 3SD from the mean from these analyses made only very minor differences to the associations observed. Associations in the BMI/SF/DXA group, independent of age, cigarette smoking, alcohol intake, and exercise habit, between adiposity measures and the key risk factors for which the strongest associations were apparent, IVGTT-Si, triglycerides, and systolic BP, are illustrated by standardised regression coefficients in Figure 1.

3.3. Blood Pressure. Associations between systolic and diastolic BP and BMI and skinfolds in the BMI/SF group were relatively weak $(R<0.20)$, the most consistent associations being seen for diastolic BP, with little differences in strength of association between the different adiposity measures (Table 2). Stronger associations were apparent in the BMI/ DXA group $(R=0.31, P<0.001$ for BMI with both systolic and diastolic BP: Table 3 ) and in the BMI/SF/DXA group $(R=0.34$ and $0.29, P<0.001$ for systolic and diastolic $\mathrm{BP}$, resp., Table 4). The strongest associations between blood pressure and adiposity measures were seen with BMI.

3.4. Lipids and Lipoproteins. In each of the three groups studied, significant associations were apparent between triglyceride concentrations and all measures of adiposity except triceps skinfold and DXA gynoid fat in the BMI/SF/DXA group (Tables 2-4). The strongest associations between triglycerides and adiposity measures were seen with BMI (BMI/SF group, $R=0.24$; BMI/DXA group, $R=0.41 ; \mathrm{BMI} / \mathrm{SF} / \mathrm{DXA}$ group, $R=0.34$, all $P<0.001)$. Similar associations were apparent between HDL cholesterol and adiposity measures, but weaker and in the opposite direction.

3.5. Insulin Sensitivity-Related Measures. In the BMI/SF group, significant negative associations were apparent between Matsuda Si and all measures of adiposity, with the strongest association being seen with BMI $(R=-0.33, P<$ 0.001 , Table 2). Similar associations were apparent between HOMA-IR, FPI, and adiposity measures, but weaker and in the opposite direction. In the BMI/DXA group significant negative associations were apparent between IVGTT-Si and all measures of adiposity, with the strongest association being seen with DXA android fat $(R=-0.47, P<0.001$, Table 3$)$. Strong associations were also seen with BMI and DXA total fat $(R=-0.42$ and -0.46 , resp., $P<0.001)$. Similar asso- 


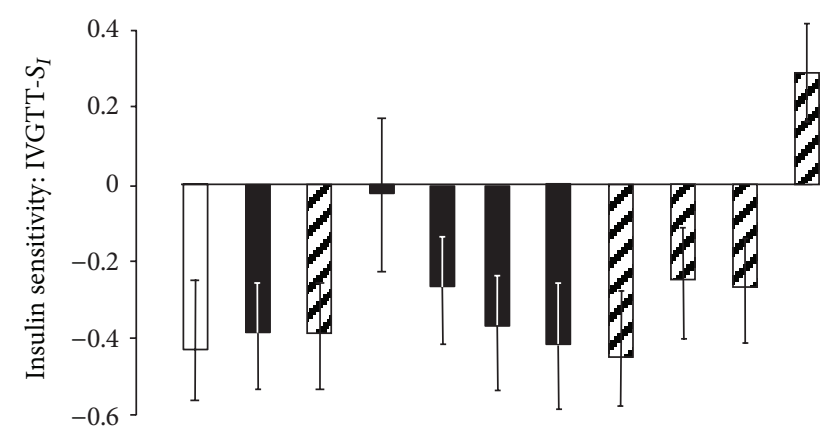

(a)

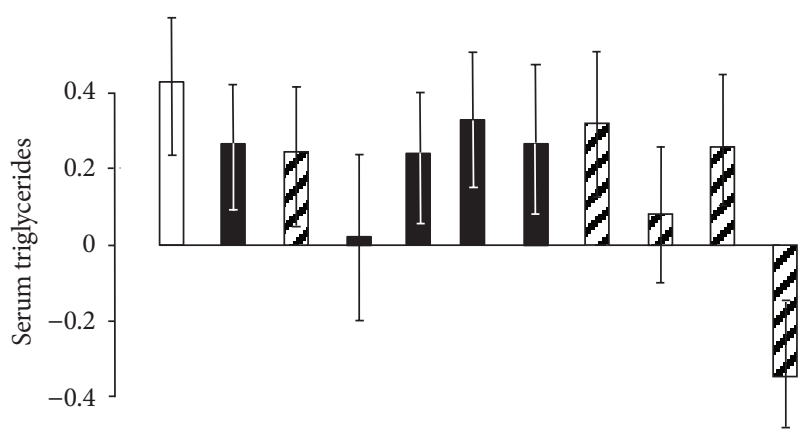

(b)

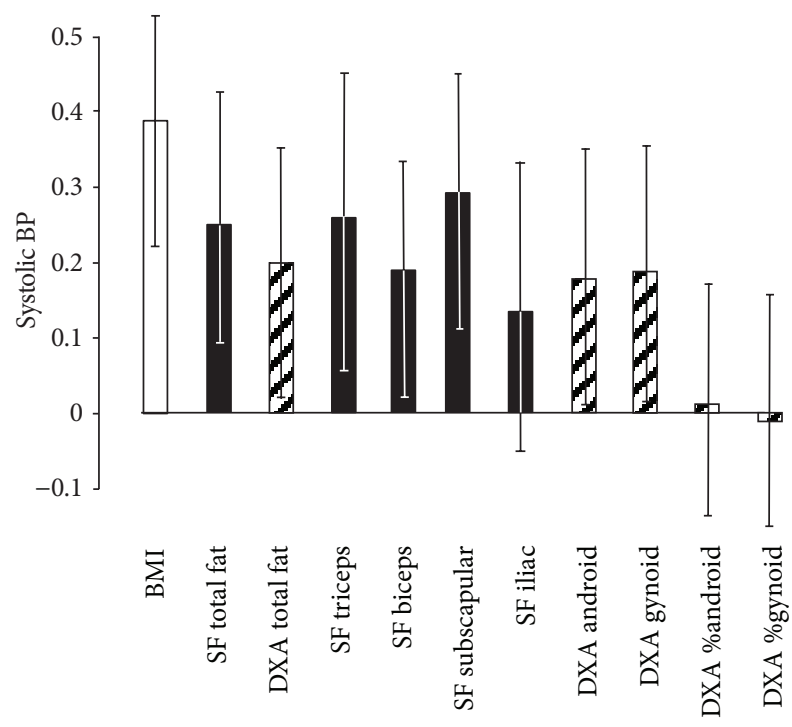

Body fat measure

(c)

Figure 1: BMI/SF/DXA $(n=156)$. Variation in insulin sensitivity, triglycerides, and systolic blood pressure with body fat measures. Regression coefficients ( $\pm 95 \%$ confidence interval) for prediction of (a) insulin sensitivity, IVGTT-Si; (b) serum triglyceride concentration; and (c) systolic blood pressure by BMI (open bars), skinfold thicknesses (closed bars), and DXA measures (hatched bars) recorded at the same visit. Standardised data was used; therefore, bars show the number of standard deviations change in IVGTT-Si, triglycerides, and systolic BP for a $1 \mathrm{SD}$ change in each body fat measure. Age, cigarette smoking, alcohol intake, and exercise habits were included in the prediction models. ciations were apparent between HOMA-IR, FPI, and adiposity measures, but weaker and in the opposite direction, and associations similar to those in the BMI/DXA group were seen in the BMI/SF/DXA group.

3.6. Inflammation-Related Measures. The only consistent associations between inflammation-related measures and measures of adiposity were between ESR and DXA total and android fat $(R=0.16-0.19, P<0.05$, Tables 3 and 4$)$.

3.7. Effects of Regional Adiposity Independent of Total Adiposity. To explore whether there was any contribution of regional body fat to risk marker variation independent of variation in total fat, each skinfold or DXA measure of regional fat was paired with its corresponding measure of total fat in partial correlation analysis. This analysis was restricted to the BMI/SF/DXA group, in which the strongest associations were seen between adiposity measures and risk markers. Associations between risk markers and SF total fat were relatively unaffected by pairing with triceps, biceps, and supra-iliac skinfold measures, each of which showed few significant associations when paired with SF total fat. There was, however, evidence of an independent contribution of the subscapular skinfold to variation triglycerides and colinearity between SF total fat and subscapular skinfold with respect to IVGTT-Si (results not shown). With inclusion of DXA android fat with DXA total fat (Table 5), both DXA total fat and DXA android fat ceased to be significantly associated with SBP, HDL cholesterol, and FPG, suggesting strong colinearity between DXA total fat and DXA android fat. There was, however, evidence of an independent effect of DXA android fat on measures of insulin sensitivity. With inclusion of DXA gynoid fat with DXA total fat (Table 5), both DXA total fat and DXA gynoid fat remained associated with variation in serum triglycerides and insulin sensitivity-related measures, but the direction of association with DXA gynoid fat was reversed, rendering its associations similar to those seen with DXA \% gynoid fat. DXA android and gynoid fat were also entered in a paired analysis of risk marker variation (Table 5). In this analysis, DXA android fat remained significantly associated with a range of risk markers, particularly serum triglycerides and insulin sensitivity-related measures, but associations between DXA gynoid fat and risk markers were eliminated for all risk markers except DBP, triglycerides, and albumin.

\section{Discussion}

Our findings confirm our hypothesis that, in this group of adult males, BMI, a simple measure of overall adiposity, can account for variation in a broad range of adiposity-related risk markers as effectively as more sophisticated measures derived from skinfold or DXA measurements. To the best of our knowledge, this is the first study to compare in a single investigation BMI, skinfolds, and DXA as correlates of risk marker variation in white adult males. One other study, that is of Steinberger and colleagues [23], combined BMI, skinfold, and DXA adiposity measures, but in 72 adolescent males and 58 adolescent females of white or black race and 
TABLE 3: BMI/DXA $(n=269)$. Partial correlation coefficients and significances between risk marker and body fat measures, independent of age, smoking, alcohol intake, and exercise habit.

\begin{tabular}{|c|c|c|c|c|c|c|}
\hline & BMI & DXA fat & DXA android & DXA gynoid & DXA $\%$ android & DXA \% gynoid \\
\hline Systolic BP & $0.31^{\S}$ & $0.22^{*}$ & $0.22^{\S}$ & $0.15^{*}$ & 0.11 & $-0.12^{*}$ \\
\hline Diastolic BP & $0.31^{\S}$ & $0.27^{\S}$ & $0.25^{\S}$ & $0.26^{\#}$ & 0.03 & -0.04 \\
\hline Triglyceride & $0.41^{\S}$ & $0.34^{\S}$ & $0.40^{\S}$ & $0.17^{\#}$ & $0.36^{\S}$ & $-0.37^{\S}$ \\
\hline HDL Cholesterol & $-0.31^{\S}$ & $-0.25^{\S}$ & $-0.27^{\S}$ & $-0.17^{\#}$ & $-0.18^{\#}$ & $0.20^{\#}$ \\
\hline FPG & $0.16^{*}$ & $0.13^{*}$ & 0.12 & 0.10 & 0.05 & -0.10 \\
\hline FPI & $0.30^{\S}$ & $0.33^{\S}$ & $0.32^{\S}$ & $0.28^{\S}$ & $0.12^{*}$ & $-0.16^{*}$ \\
\hline HOMA-IR & $0.31^{\S}$ & $0.33^{\S}$ & $0.32^{\S}$ & $0.28^{\S}$ & $0.12^{*}$ & $-0.16^{\#}$ \\
\hline IVGTT-Si & $-0.42^{\S}$ & $-0.46^{\S}$ & $-0.47^{\S}$ & $-0.33^{\S}$ & $-0.27^{\S}$ & $0.30^{\S}$ \\
\hline ESR & 0.12 & $0.16^{*}$ & $0.16^{*}$ & 0.12 & 0.05 & -0.07 \\
\hline
\end{tabular}

Significances: ${ }^{*} P<0.05 ;{ }^{\#} P<0.01 ;{ }^{\lessgtr} P<0.001$.

DXA: dual-energy X-ray absorptiometry; SF: skinfold thickness.

with insulin sensitivity being measured by the euglycaemic hyperinsulinaemic clamp. Despite these differences, their conclusions agree with our finding that BMI is as effective at accounting for risk marker variation as skinfold or DXAderived measures.

Other studies, that have included DXA but not skinfold thickness measurements, have reported little difference in strength of association between simple anthropometric and DXA measures of adiposity with respect to risk marker variation [24-28], and there is also evidence that this is the case with regard to studies including BMI and skinfold but not DXA measurements [29, 30]. Moreover, there is even evidence that risk marker associations with simple, anthropometric measures of adiposity can be comparable to those with specific fat depots quantified by magnetic resonance imaging (MRI) [31].

Nevertheless, a number of other studies in which specific fat depots have been measured by MRI or computed tomography have raised the possibility that the visceral fat depot, with its circulation draining into the hepatic portal vein, is particularly associated with risk marker variation [32-34]. It has been proposed that variation specifically in the visceral fat depot can account for discrepancies in associations between risk markers and overall adiposity, with some obese individuals being metabolically healthy and some individuals with apparently normal levels of adiposity exhibiting a metabolic phenotype typical of obesity [35]. However, a number of other studies have found risk marker variation to be equally associated with variation in visceral or subcutaneous fat depots [36-40]. The relative importance of the visceral and subcutaneous fat depots in risk marker variation could vary according to age, race, or gender and may be affected by variation in the strengths of relationship according to degree of overweight, with the visceral fat depot assuming more importance in overt obesity [41, 42].

Our study has limitations and strengths. Recruitment was in the context of a long-running, open cohort study and the participants and the periods during which data was being collected differed between the three groups studied. Consequently, results were based on three cross-sectional samples, which differed in demographic and risk factor characteristics. Findings should, therefore, be interpreted with caution. It should be noted, however, that relative strengths of association between measures of adiposity and risk factors were generally comparable between crosssections, which lends support to their validity.

A further potential limitation of our study was that waist and hip circumferences were not measured. Various anthropometric measures of centrally concentrated adiposity, including waist circumference, waist hip ratio, and waist height ratio, have been explored in previous studies in relation to clinical outcomes and have generally been found to predict clinical outcomes more strongly than BMI $[9,43]$, although the differential in improvement may be small $[8$, 44]. It is questionable, however, whether anthropometric measures of centrality would have provided any more information than that provided by the DXA measurements of android fat included in our study. Nevertheless, given that central fat measures are more predictive of clinical outcomes and that the central fat measure in our study showed little difference from BMI in the strengths of its relationships with the risk factors we measured, it may be inferred that there are factors associated with variation in central fat that we did not measure but which impact strongly on clinical outcomes $[45,46]$. A particular advantage of our analysis was the relatively low use of medications likely to affect risk marker variation, particularly use of lipid-lowering agents, the majority of measurements having been carried out before the inception of the current, widespread use of these agents. A further advantage was the broad range of risk markers available for analysis, which allowed for strong confirmation of the relative strengths of association between risk markers and adiposity measures.

The three indices of total body fat, BMI, SF total fat, and DXA total fat were closely correlated, as was subscapular skinfold with SF total fat and DXA android and gynoid with DXA total fat. Risk marker associations suggested that subscapular skinfold alone might predict risk marker variation as effectively as a measure of total adiposity derived from all four skinfolds. As mentioned, DXA android fat conveyed as much if not more information in this respect as did DXA total fat and risk marker associations with DXA 


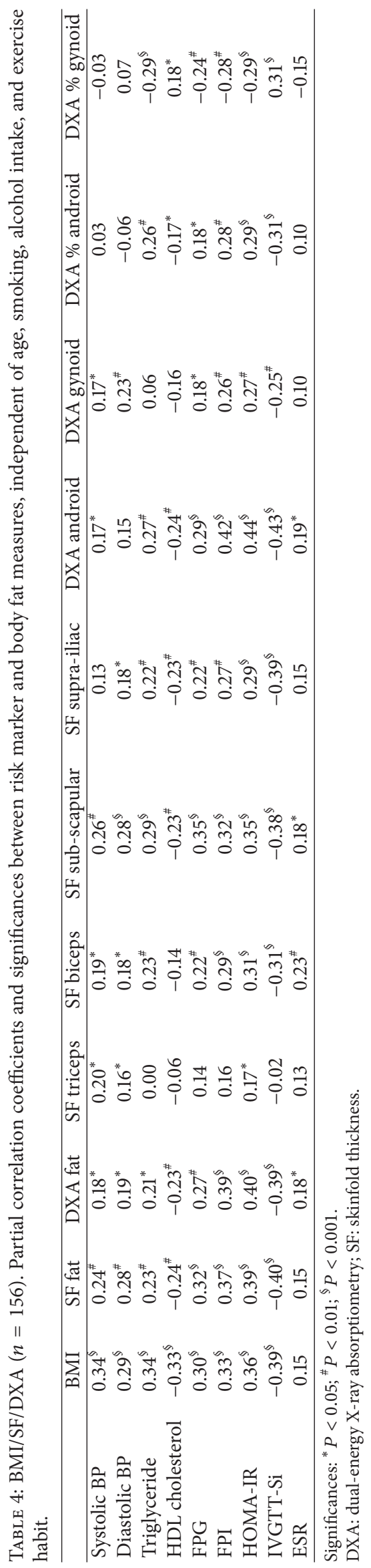


TABLE 5: Partial correlation coefficients between risk marker measures and paired DXA measures $(n=156)$. Three correlation analyses were explored: Analysis 1: risk factor versus DXA total, DXA android, age, smoking, alcohol intake and exercise habit.Analysis 2: risk factor versus DXA total, DXA gynoid, age, smoking, alcohol intake and exercise habit.Analysis 3: risk factor versus DXA android, DXA gynoid, age, smoking, alcohol intake and exercise habit.

\begin{tabular}{|c|c|c|c|c|c|c|}
\hline & \multicolumn{2}{|c|}{ Analysis 1} & \multicolumn{2}{|c|}{ Analysis 2} & \multicolumn{2}{|c|}{ Analysis 3} \\
\hline & DXA total & DXA android & DXA total & DXA gynoid & DXA android & DXA gynoid \\
\hline Systolic BP & 0.08 & -0.02 & 0.06 & 0.02 & 0.06 & 0.07 \\
\hline Diastolic BP & $0.17^{*}$ & -0.12 & -0.03 & 0.14 & -0.03 & $0.19^{*}$ \\
\hline Total Cholesterol & -0.06 & 0.07 & 0.10 & -0.11 & 0.08 & -0.09 \\
\hline LDL Cholesterol & 0.00 & 0.00 & 0.02 & -0.02 & 0.01 & -0.01 \\
\hline Triglyceride & -0.16 & $0.23^{\#}$ & $0.32^{\S}$ & $-0.26^{\#}$ & $0.32^{\S}$ & $-0.19^{*}$ \\
\hline HDL Cholesterol & 0.03 & -0.10 & $-0.18^{*}$ & 0.09 & $-0.19^{*}$ & 0.03 \\
\hline FPG & 0.00 & 0.08 & $0.24^{\#}$ & -0.13 & $0.23^{\#}$ & -0.04 \\
\hline FPI & -0.06 & $0.19^{*}$ & $0.34^{\S}$ & $-0.18^{*}$ & $0.35^{\S}$ & -0.07 \\
\hline HOMA-IR & -0.05 & $0.19^{*}$ & $0.36^{\S}$ & $-0.19^{*}$ & $0.36^{\S}$ & -0.07 \\
\hline IVGTT-Si & 0.08 & $-0.22^{\#}$ & $-0.37^{\S}$ & $0.22^{\#}$ & $-0.38^{\S}$ & 0.10 \\
\hline white cell count & 0.04 & -0.02 & 0.12 & -0.10 & 0.07 & -0.04 \\
\hline ESR & 0.00 & 0.06 & $0.21^{*}$ & -0.14 & $0.18^{*}$ & -0.06 \\
\hline globulin & 0.01 & -0.03 & -0.07 & 0.05 & -0.06 & 0.02 \\
\hline albumin & -0.13 & 0.16 & $0.22^{\#}$ & $-0.20^{*}$ & $0.21^{*}$ & $-0.17^{*}$ \\
\hline
\end{tabular}

Significances: ${ }^{*} P<0.05 ;{ }^{\#} P<0.01 ;{ }^{\S} P<0.001$.

DXA: dual-energy X-ray absorptiometry; SF: skinfold thickness.

$\%$ android followed closely on those with DXA android fat. However, the pattern of associations with DXA gynoid fat was markedly different in that DXA \% gynoid fat showed opposite directions of association with risk markers to those seen with DXA gynoid fat mass. The strong positive association between DXA gynoid and DXA total fat masses suggests that the DXA gynoid measure incorporates a strong component of variation that simply reflects net adiposity and this could account for the unfavourable associations seen between risk markers and DXA gynoid fat. Rendering DXA gynoid as a percentage of body fat, however, distinguished variation in gynoid fat from variation in total fat. As a result of making this distinction, it was apparent that there may be a component of variation in gynoid or lower body fat that has a favourable effect on risk marker variation, as has been suggested by previous studies [47-51]. However, DXA \% gynoid fat was also strongly, inversely, associated with DXA \% android fat, and it could be argued that a high percentage of gynoid fat merely reflects a low percentage of android fat (and vice versa); the favourable associations seen between risk markers and DXA \% gynoid are then merely secondary to variation in android fat. The analyses we present in Table 5, in which risk marker associations with DXA gynoid fat largely disappeared with inclusion of DXA android fat, which supports the possibility that the favourable associations seen between risk markers and DXA \% gynoid are merely secondary to variation in android fat. If DXA gynoid fat had been having an underlying, independent, favourable effect on risk marker variation, these favourable associations would have been expected to remain despite inclusion of android fat in the analysis.

Overall, the strongest associations seen with variation in any measure of adiposity were with measures of insulin sensitivity, followed by serum triglyceride, HDL cholesterol concentrations, and then blood pressure. Serum cholesterol and LDL cholesterol concentrations varied inconsistently with adiposity $[52,53]$. Consistent relationships between adiposity and indices of inflammation were restricted to positive associations between DXA total and android fat and ESR. In contrast to our findings, significant associations between white cell count and BMI have been reported [54], but their magnitude can be relatively small [55]. Adiposityrelated inflammation relates to macrophage infiltration and activation and is associated with elevated levels of specific cytokines including interleukin-6, tumour necrosis factoralpha, and acute phase proteins such as C-reactive protein [6]. It is possible that, had we measured these, stronger associations would have been apparent. It is, nevertheless, noteworthy that in this cohort the inflammation markers we evaluated are significantly related to clinical outcomes $[56,57]$.

In conclusion, BMI can account for variation in risk markers in white, predominantly middle-aged males as well as more sophisticated measures derived from skinfold thickness measurements or DXA scanning.

\section{Conflict of Interests}

The study received financial support from the Heart disease and Diabetes Research Trust, the Atherosclerosis Research Trust, and the Rosen Foundation. Funding agencies had no involvement in the study or its reporting. The authors report no declarations of interest.

\section{Acknowledgments}

HDDRISC study was initiated by the late Professor Victor Wynn, who directed it for much of its course. Since the 
HDDRISC study began, it has been sustained by many clinical, scientific, technical, nursing, and administrative staff, to each of whom the authors would like to extend their thanks.

\section{References}

[1] U. Schorr, K. Blaschke, S. Turan, A. Distler, and A. M. Sharma, "Relationship between angiotensinogen, leptin and blood pressure levels in young normotensive men," Journal of Hypertension, vol. 16, no. 10, pp. 1475-1480, 1998.

[2] V. Mohamed-Ali, J. H. Pinkney, and S. W. Coppack, "Adipose tissue as an endocrine and paracrine organ," International Journal of Obesity, vol. 22, no. 12, pp. 1145-1158, 1998.

[3] S. W. Coppack, T. J. Yost, R. M. Fisher, R. H. Eckel, and J. M. Miles, "Periprandial systemic and regional lipase activity in normal humans," The American Journal of Physiology-Endocrinology and Metabolism, vol. 270, no. 4, pp. E718-E722, 1996.

[4] G. S. Hotamisligil, N. S. Shargill, and B. M. Spiegelman, "Adipose expression of tumor necrosis factor- $\alpha$ : direct role in obesity-linked insulin resistance," Science, vol. 259, no. 5091, pp. 87-91, 1993.

[5] J. M. L. Stouthard, R. P. J. O. Elferink, and H. P. Sauerwein, "Interleukin-6 enhances glucose transport in 3T3-L1 adipocytes," Biochemical and Biophysical Research Communications, vol. 220, no. 2, pp. 241-245, 1996.

[6] C. R. Caruso, C. R. Balistreri, and G. Candore, "The role of adipose tissue and adipokines in obesity-related inflammatory diseases," Mediators of Inflammation, vol. 2010, Article ID 802078, 19 pages, 2010.

[7] E. B. Rimm, M. J. Stampfer, E. Giovannucci et al., "Body size and fat distribution as predictors of coronary heart disease among middle-aged and older US men," The American Journal of Epidemiology, vol. 141, no. 12, pp. 1117-1127, 1995.

[8] K. M. Rexrode, J. E. Buring, and J. E. Manson, "Abdominal and total adiposity and risk of coronary heart disease in men," International Journal of Obesity, vol. 25, no. 7, pp. 1047-1056, 2001.

[9] H. J. Schneider, N. Friedrich, J. Klotsche et al., "The predictive value of different measures of obesity for incident cardiovascular events and mortality," Journal of Clinical Endocrinology and Metabolism, vol. 95, no. 4, pp. 1777-1785, 2010.

[10] P. Björntorp, "Portal' adipose tissue as a generator of risk factors for cardiovascular disease and diabetes," Arteriosclerosis, Thrombosis, and Vascular Biology, vol. 10, pp. 493-496, 1990.

[11] J. Jaspan and K. Polonsky, "Glucose ingestion in dogs alters the hepatic extraction of insulin. In vivo evidence for a relationship between biologic action and extraction of insulin," Journal of Clinical Investigation, vol. 69, no. 3, pp. 516-525, 1982.

[12] R. Malmström, C. J. Packard, M. Caslake et al., "Defective regulation of triglyceride metabolism by insulin in the liver in NIDDM," Diabetologia, vol. 40, no. 4, pp. 454-462, 1997.

[13] J. V. G. A. Durnin and J. Womersley, "Body fat assessed from total body density and its estimation from skinfold thickness: measurements on 481 men and women aged from 16 to 72 years," The British Journal of Nutrition, vol. 32, no. 1, pp. 79-97, 1974.

[14] O. L. Svendsen, J. Haarbo, C. Hassager, and C. Christiansen, "Accuracy of measurements of body composition by dualenergy X-ray absorptiometry in vivo," The American Journal of Clinical Nutrition, vol. 57, no. 5, pp. 605-608, 1993.
[15] R. B. Mazess, H. S. Barden, J. P. Bisek, and J. Hanson, "Dualenergy X-ray absorptiometry for total-body and regional bonemineral and soft-tissue composition," The American Journal of Clinical Nutrition, vol. 51, no. 6, pp. 1106-1112, 1990.

[16] I. F. Godsland, O. F. Agbaje, and R. Hovorka, "Evaluation of nonlinear regression approaches to estimation of insulin sensitivity by the minimal model with reference to Bayesian hierarchical analysis," The American Journal of Physiology-Endocrinology and Metabolism, vol. 291, no. 1, pp. E167-E174, 2006.

[17] I. F. Godsland, F. Leyva, C. Walton, M. Worthington, and J. C. Stevenson, "Associations of smoking, alcohol and physical activity with risk factors for coronary heart disease and diabetes in the first follow-up cohort of the heart disease and diabetes risk indicators in a screened cohort study (HDDRISC-1)," Journal of Internal Medicine, vol. 244, no. 1, pp. 33-41, 1998.

[18] C. J. Ley, B. Lees, and J. C. Stevenson, "Sex- and menopauseassociated changes in body-fat distribution," The American Journal of Clinical Nutrition, vol. 55, no. 5, pp. 950-954, 1992.

[19] W. T. Friedewald, R. I. Levy, and D. S. Fredrickson, "Estimation of the concentration of low-density lipoprotein cholesterol in plasma, without use of the preparative ultracentrifuge," Clinical Chemistry, vol. 18, no. 6, pp. 499-502, 1972.

[20] D. R. Matthews, J. P. Hosker, A. S. Rudenski, B. A. Naylor, D. F. Treacher, and R. C. Turner, "Homeostasis model assessment: insulin resistance and $\beta$-cell function from fasting plasma glucose and insulin concentrations in man," Diabetologia, vol. 28, no. 7, pp. 412-419, 1985.

[21] M. Matsuda and R. A. DeFronzo, "Insulin sensitivity indices obtained from oral glucose tolerance testing: comparison with the euglycemic insulin clamp," Diabetes Care, vol. 22, no. 9, pp. 1462-1470, 1999.

[22] T. V. Perneger, "What's wrong with Bonferroni adjustments," The British Medical Journal, vol. 316, no. 7139, pp. 1236-1238, 1998.

[23] J. Steinberger, D. R. Jacobs Jr., S. Raatz, A. Moran, C. P. Hong, and A. R. Sinaiko, "Comparison of body fatness measurements by BMI and skinfolds vs dual energy X-ray absorptiometry and their relation to cardiovascular risk factors in adolescents," International Journal of Obesity, vol. 29, no. 11, pp. 1346-1352, 2005.

[24] H. Ito, K. Nakasuga, A. Ohshima et al., "Detection of cardiovascular risk factors by indices of obesity obtained from anthropometry and dual-energy X-ray absorptiometry in Japanese individuals," International Journal of Obesity, vol. 27, no. 2, pp. 232-237, 2003.

[25] J. Sierra-Johnson, B. D. Johnson, K. R. Bailey, and S. T. Turner, "Relationships between insulin sensitivity and measures of body fat in asymptomatic men and women," Obesity Research, vol. 12, no. 12, pp. 2070-2077, 2004.

[26] K. Lee, Y. M. Song, and J. Sung, "Which obesity indicators are better predictors of metabolic risk? Healthy twin study," Obesity, vol. 16, no. 4, pp. 834-840, 2008.

[27] E. Hemmingsson, J. Uddén, and M. Neovius, "No apparent progress in bioelectrical impedance accuracy: validation against metabolic risk and DXA," Obesity, vol. 17, no. 1, pp. 183-187, 2009.

[28] Q. Sun, R. M. van Dam, D. Spiegelman, S. B. Heymsfield, W. C. Willett, and F. B. Hu, "Comparison of dual-energy X-ray abso- 
rptiometric and anthropometric measures of adiposity in relation to adiposity-related biologic factors," The American Journal of Epidemiology, vol. 172, no. 12, pp. 1442-1454, 2010.

[29] D. Spiegelman, R. G. Israel, C. Bouchard, and W. C. Willett, "Absolute fat mass, percent body fat, and body-fat distribution: which is the real determinant of blood pressure and serum glucose?" The American Journal of Clinical Nutrition, vol. 55, no. 6, pp. 1033-1044, 1992.

[30] D. S. Freedman, P. T. Katzmarzyk, W. H. Dietz, S. R. Srinivasan, and G. S. Berenson, "The relation of BMI and skinfold thicknesses to risk factors among young and middle-aged adults: the Bogalusa heart study," Annals of Human Biology, vol. 37, no. 6, pp. 726-737, 2010.

[31] R. Scherzer, W. Shen, P. Bacchetti et al., "Simple anthropometric measures correlate with metabolic risk indicators as strongly as magnetic resonance imaging-measured adipose tissue depots in both HIV-infected and control subjects," The American Journal of Clinical Nutrition, vol. 87, no. 6, pp. 1809-1817, 2008.

[32] C. S. Fox, J. M. Massaro, U. Hoffmann et al., "Abdominal visceral and subcutaneous adipose tissue compartments: association with metabolic risk factors in the Framingham heart study," Circulation, vol. 116, no. 1, pp. 39-48, 2007.

[33] M. Piché, A. Lapointe, S. J. Weisnagel et al., "Regional body fat distribution and metabolic profile in postmenopausal women," Metabolism, vol. 57, no. 8, pp. 1101-1107, 2008.

[34] J. D. Smith, A. L. Borel, J. A. Nazare et al., "Visceral adipose tissue indicates the severity of cardiometabolic risk in patients with and without type 2 diabetes: results from the INSPIRE ME IAA study," Journal of Clinical Endocrinology and Metabolism, vol. 97, no. 5, pp. 1517-1525, 2012.

[35] E. L. Thomas, G. Frost, S. D. Taylor-Robinson, and J. D. Bell, "Excess body fat in obese and normal-weight subjects," Nutrition Research Reviews, vol. 25, no. 1, pp. 150-161, 2012.

[36] N. Abate, A. Garg, R. M. Peshock, J. Stray-Gundersen, and S. M. Grundy, "Relationships of generalized and regional adiposity to insulin sensitivity in men," Journal of Clinical Investigation, vol. 96, no. 1, pp. 88-98, 1995.

[37] B. H. Goodpaster, F. L. Thaete, J. Simoneau, and D. E. Kelley, "Subcutaneous abdominal fat and thigh muscle composition predict insulin sensitivity independently of visceral fat," Diabetes, vol. 46, no. 10, pp. 1579-1585, 1997.

[38] Y. Miyazaki, L. Glass, C. Triplitt, E. Wajcberg, L. J. Mandarino, and R. A. DeFronzo, "Abdominal fat distribution and peripheral and hepatic insulin resistance in type 2 diabetes mellitus," The American Journal of Physiology-Endocrinology and Metabolism, vol. 283, no. 6, pp. E1135-E1143, 2002.

[39] D. E. Laaksonen, S. Kainulainen, A. Rissanen, and L. Niskanen, "Relationships between changes in abdominal fat distribution and insulin sensitivity during a very low calorie diet in abdominally obese men and women," Nutrition, Metabolism and Cardiovascular Diseases, vol. 13, no. 6, pp. 349-356, 2003.

[40] S. M. Kang, J. W. Yoon, H. Y. Ahn et al., "Android fat depot is more closely associated with metabolic syndrome than abdominal visceral fat in elderly people," PLOS ONE, vol. 6, no. 11, Article ID e27694, 2011.

[41] E. Bonora, "Relationship between regional fat distribution and insulin resistance," International Journal of Obesity, vol. 24, supplement 2, pp. S32-S35, 2000.
[42] S. K. Gan, A. D. Kriketos, A. M. Poynten et al., "Insulin action, regional fat, and myocyte lipid: altered relationships with increased adiposity," Obesity Research, vol. 11, no. 11, pp. 1295-1305, 2003.

[43] B. Larsson, K. Svärdsudd, L. Welin, L. Wilhelmsen, P. Björntorp, and G. Tibblin, "Abdominal adipose tissue distribution, obesity, and risk of cardiovascular disease and death: 13 year follow up of participants in the study of men born in 1913," The British Medical Journal, vol. 288, pp. 1401-1404, 1984.

[44] G. Hu, J. Tuomilehto, K. Silventoinen, N. Barengo, and P. Jousilahti, "Joint effects of physical activity, body mass index, waist circumference and waist-to-hip ratio with the risk of cardiovascular disease among middle-aged Finnish men and women," European Heart Journal, vol. 25, no. 24, pp. 2212-2219, 2004.

[45] E. L. Thomas, J. R. Parkinson, G. S. Frost et al., “The missing risk: MRI and MRS phenotyping of abdominal adiposity and ectopic fat," Obesity, vol. 20, no. 1, pp. 76-87, 2012.

[46] A. J. Cameron, D. J. Magliano, J. E. Shaw et al., "The influence of hip circumference on the relationship between abdominal obesity and mortality," International Journal of Epidemiology, vol. 41, no. 2, Article ID dyr198, pp. 484-494, 2012.

[47] R. B. Terry, M. L. Stefanick, W. L. Haskell, and P. D. Wood, "Contributions of regional adipose tissue depots to plasma lipoprotein concentrations in overweight men and women: possible protective effects of thigh fat," Metabolism, vol. 40, no. 7, pp. 733-740, 1991.

[48] J. C. Seidell, L. Pérusse, J.-. Després, and C. Bouchard, "Waist and hip circumferences have independent and opposite effects on cardiovascular disease risk factors: the Quebec family study," The American Journal of Clinical Nutrition, vol. 74, no. 3, pp. 315321, 2001.

[49] M. B. Snijder, J. M. Dekker, M. Visser et al., “Trunk fat and leg fat have independent and opposite associations with fasting and postload glucose levels: the hoorn study," Diabetes Care, vol. 27, no. 2, pp. 372-377, 2004.

[50] H. Wu, Q. Qi, Z. Yu et al., "Independent and opposite associations of trunk and leg fat depots with adipokines, inflammatory markers, and metabolic syndrome in middle-aged and older Chinese men and women," Journal of Clinical Endocrinology and Metabolism, vol. 95, no. 9, pp. 4389-4398, 2010.

[51] K. N. Manolopoulos, F. Karpe, and K. N. Frayn, "Gluteofemoral body fat as a determinant of metabolic health," International Journal of Obesity, vol. 34, no. 6, pp. 949-959, 2010.

[52] H. S. Park, J. Y. Park, and R. Yu, "Relationship of obesity and visceral adiposity with serum concentrations of CRP, TNF- $\alpha$ and IL-6," Diabetes Research and Clinical Practice, vol. 69, no. 1, pp. 29-35, 2005.

[53] S. A. Lear, M. M. Chen, C. L. Birmingham, and J. J. Frohlich, "The relationship between simple anthropometric indices and C-reactive protein: ethnic and gender differences," Metabolism, vol. 52, no. 12, pp. 1542-1546, 2003.

[54] C. A. Wilson, G. Bekele, M. Nicolson, E. Ravussin, and R. E. Pratley, "Relationship of the white blood cell count to body fat: role of leptin," The British Journal of Haematology, vol. 99, no. 2, pp. 447-451, 1997.

[55] J. B. Dixon and P. E. O’Brien, "Obesity and the white blood cell count: changes with sustained weight loss," Obesity Surgery, vol. 16, no. 3, pp. 251-257, 2006. 
[56] I. F. Godsland, R. Bruce, J. A. R. Jeffs, F. Leyva, C. Walton, and J. C. Stevenson, "Inflammation markers and erythrocyte sedimentation rate but not metabolic syndrome factor score predict coronary heart disease in high socioeconomic class males: the HDDRISC study," International Journal of Cardiology, vol. 97, no. 3, pp. 543-550, 2004.

[57] I. F. Godsland, B. V. North, and D. G. Johnston, "Simple indices of inflammation as predictors of death from cancer or cardiovascular disease in a prospective cohort after two decades of follow-up," Quarterly Journal of Medicine, vol. 104, no. 5, pp. 387-394, 2011. 


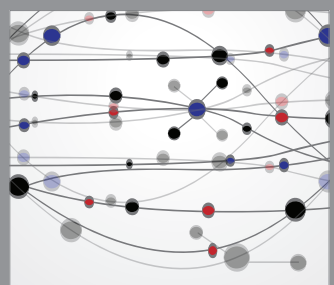

The Scientific World Journal
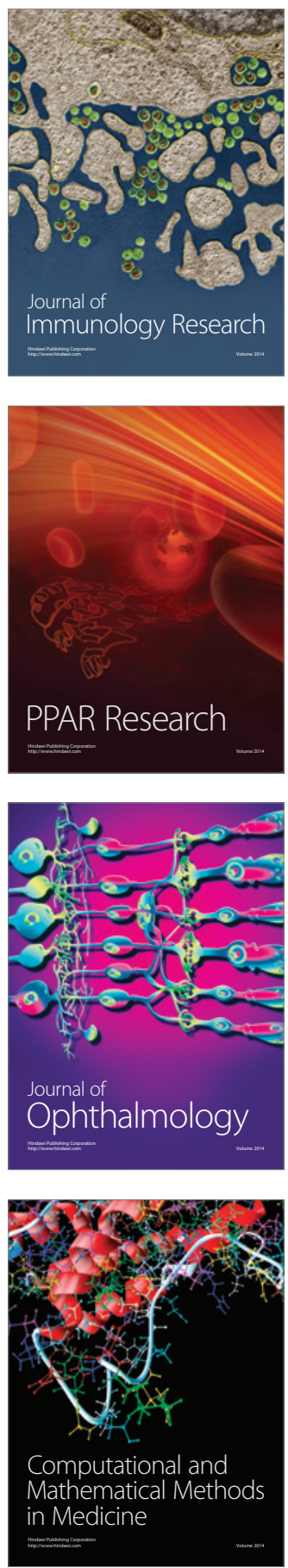

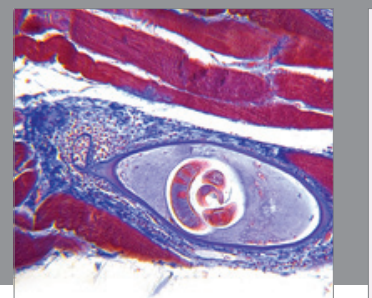

Gastroenterology

Research and Practice
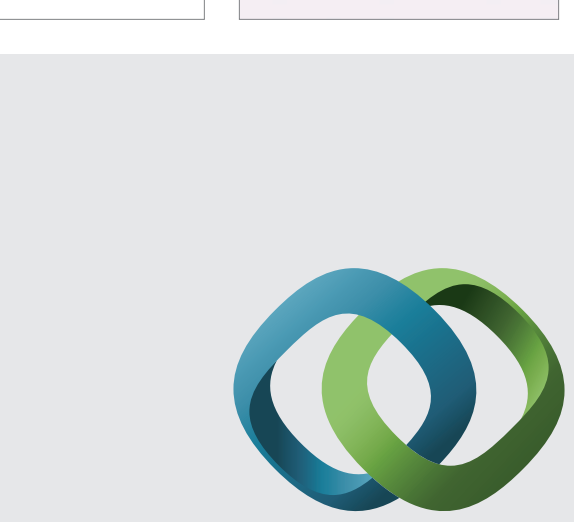

\section{Hindawi}

Submit your manuscripts at

http://www.hindawi.com
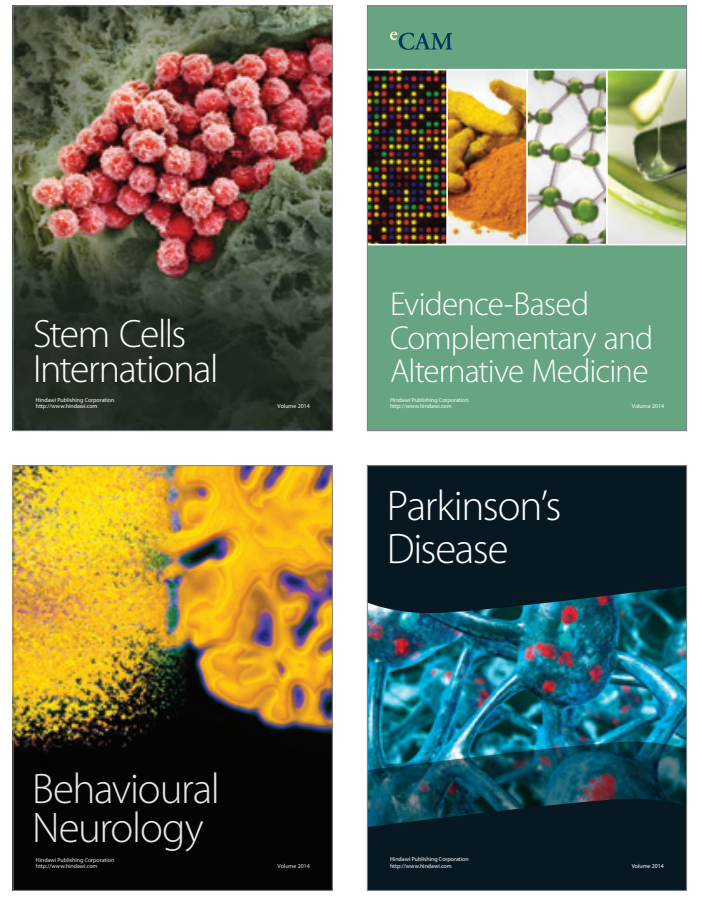
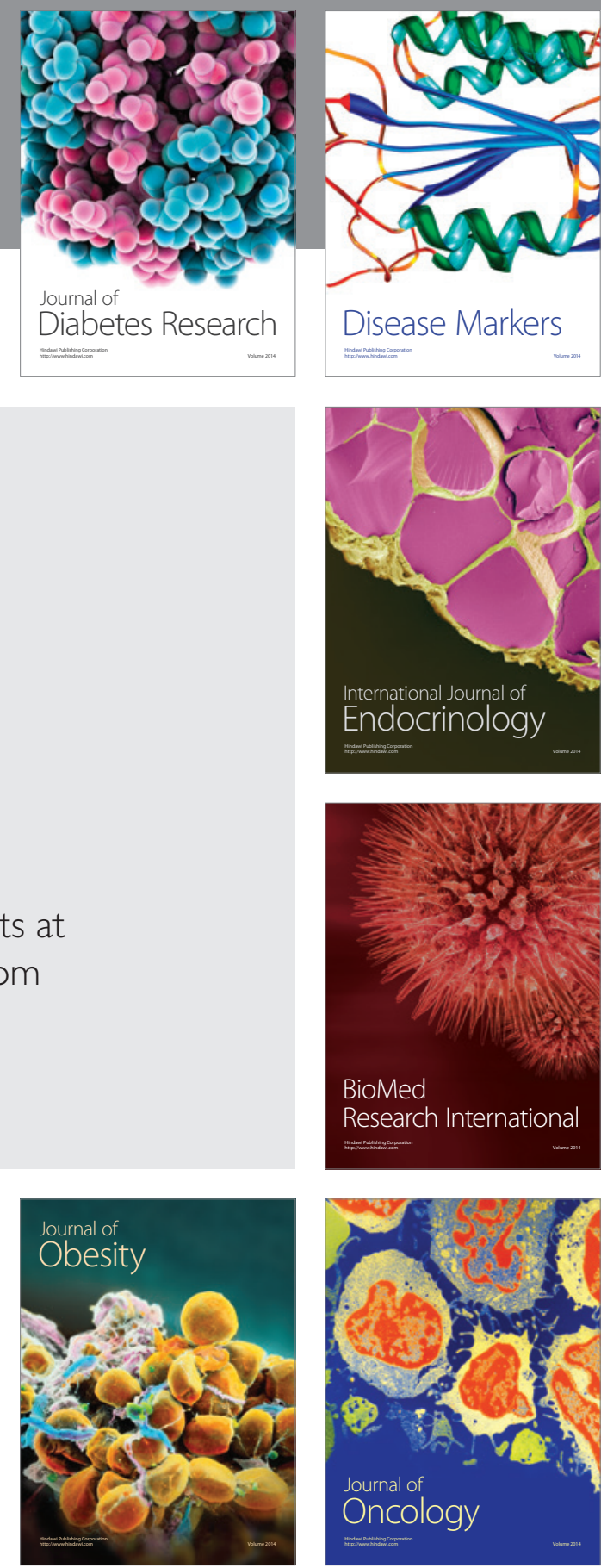

Disease Markers
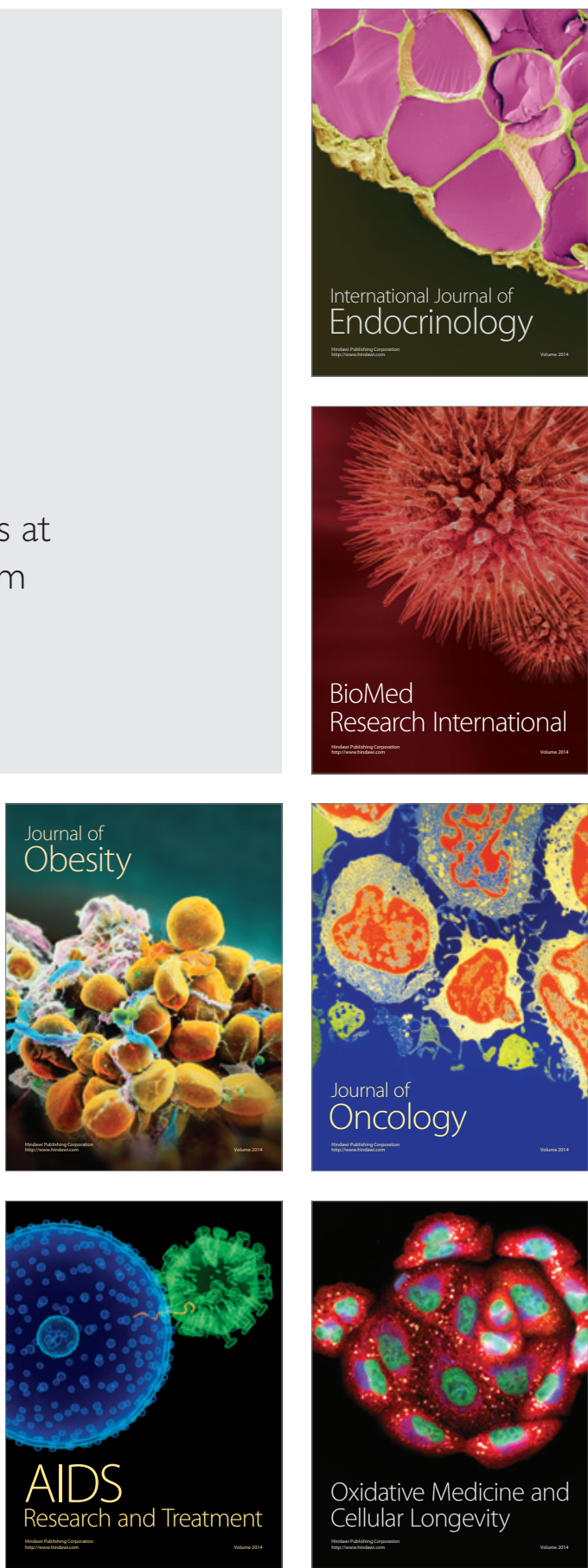